\title{
QUADRATIC FORMS AND SIMILARITIES
}

BY DANIEL B. SHAPIRO

Communicated by Hyman Bass, May 5, 1975

1. Introduction. The results announced here are concerned with the Hurwitz problem of composition of quadratic forms, over a field $F$ characteristic not two. The possible dimensions of forms admitting composition are stated. However, determining which quadratic forms do admit composition is a more delicate question, and answers are known only for small dimensions. The proofs will appear elsewhere.

It is a pleasure to acknowledge the interest and encouragement of T.-Y. Lam, A. Wadsworth, and A. Geramita in this research.

2. The Hurwitz problem. We follow the notation of Lam's book [L]. Throughout this paper, $F$ will denote a field, with char $F \neq 2$. All forms considered will be nonsingular.

Definition. If $(V, q)$ is a quadratic space over $F$, a map $f \in \operatorname{End}(V)$ is a similarity if

$$
q(f(v))=\sigma(f) \cdot q(v), \quad \text { for all } v \in V,
$$

where $\sigma(f) \in F$. Let $\operatorname{Sim}(V, q)=\operatorname{Sim}(q)$ denote the set of all similarities on $(V, q)$.

We are interested in the additive structure if $\operatorname{Sim}(V, q)$. If $S$ is an $F$. linear subspace of $\operatorname{End}(V)$, and $S \subseteq \operatorname{Sim}(V, q)$, then the map $\sigma: \operatorname{Sim}(V, q) \rightarrow$ $F$ becomes a quadratic form when restricted to $S$. We consider only those subspaces $S$ on which this form is nonsingular.

Notation. For quadratic forms $\sigma, q$, we write $\sigma<\operatorname{Sim}(q)$ if $\sigma$ is isometric to some subspace of $\operatorname{Sim}(q)$, using the induced quadratic form.

Using the definition of composition of quadratic forms in [L, p. 133], we see that $q$ admits composition with $\sigma$ if and only if $\sigma<\operatorname{Sim}(q)$. The study of such composition began with the four and eight square problem, and was completed by Hurwitz in the case when $F$ is algebraically closed $[\mathbf{H}]$.

Notation. Following [L], [K2], for $a_{i} \in F$, we write $\left\langle a_{1}, \ldots, a_{n}\right\rangle$ for an $n$-dimensional diagonal form; and $\left\langle\left\langle a_{1}, \ldots, a_{n}\right\rangle\right.$ for the $n$-fold Pfister form $\bigotimes_{i=1}^{n}\left\langle 1, a_{i}\right\rangle$. For quadratic forms $\varphi, q$, we write $\varphi \simeq q$ if they are isometric,

AMS (MOS) subject classifications (1970). Primary 15 A63, 15 A66.

Key words and phrases. Quadratic form, Hurwitz problem, similarity, Pfister form, orthogonal design. 
and $\varphi<q$ if $\varphi$ is isometric to a subform of $q$. We write $\varphi / q$, and say that $\varphi$ is a factor of $q$, if there exists a form $\tau$ with $q \simeq \varphi \otimes \tau$.

Proposition 1. Let $q$ be a quadratic form, $a, b \in F$.

(i) $\langle 1, a\rangle<\operatorname{Sim}(q)$ if and only if $\langle\langle a\rangle\rangle \mid q$.

(ii) $\langle 1, a, b\rangle<\operatorname{Sim}(q)$ if and only if $\langle\langle a, b\rangle\rangle \mid q$.

Corresponding results for larger subspaces fail, but there is a generalization in the anisotropic case in Theorem 4.

We get a good upper bound on the dimensions of $\operatorname{subspaces}$ of $\operatorname{Sim}(q)$ by tensoring up to the algebraic closure of $F$ and applying the arguments of $[\mathbf{H}]$.

Definition OF THE HuRwitz FUnCtion: If $n=2^{r} \cdot t$, where $t$ is odd and $r=4 a+b, 0 \leqslant b \leqslant 3$, then let $\rho(n)=8 a+2^{b}$.

THEOREM 2. Suppose $\sigma$ and $q$ are quadratic forms, and $\operatorname{dim} q=n$. If $\sigma$ $<\operatorname{Sim}(q)$, then $\operatorname{dim} \sigma \leqslant \rho(n)$.

Since composition algebras over $F$ exist $[\mathbf{J}]$, this bound is achieved if $q$ is an $r$-fold Pfister form, and $r \leqslant 3$. In fact, it is achieved for every Pfister form.

THEOREM 3. If $q$ is a quadratic form, $\operatorname{dim} q=n$, and $q \simeq \varphi \otimes \tau$, where $\varphi$ is Pfister and $\operatorname{dim} \tau$ is odd, then there exists a form $\sigma$ with $\sigma<\operatorname{Sim}(q)$ and $\operatorname{dim} \sigma=\rho(n)$.

There are two different constructions of such forms $\sigma$. One is a tensor product method, originally given in [G1], [G2]. The other construction is obtained from a natural Clifford algebra structure on the underlying space of the Pfister form $\varphi$ by suitably twisting the multiplication. In the 3-fold case, we get a new, close relationship between Clifford and Cayley algebras.

We can also consider $\operatorname{Sim}(V, B)$ when $B$ is a skew-symmetric form on $V$ and ask the same questions. More generally, suppose $K$ is a skew field with involution, where $F$ is the fixed field of the involution. Then $\operatorname{Sim}_{K}(V, h)$ can be defined for a $\lambda$-hermitian space $(V, h)$ (where $\lambda= \pm 1$ ). If $f \in \operatorname{Sim}_{K}(V, h)$, then $\sigma(f) \in F$, so that the subspaces of similarities are still $F$-quadratic spaces. When $K$ is a field, and when $K$ is a quaternion division algebra, the sharp upper bound functions for dimensions are known. (Compare [W, p. 438].) These functions are closely related to the Hurwitz function $\rho(n)$.

3. Pfister factors. Proposition 1 concerns the relationship between subspaces of similarities and Pfister factors. It can be generalized in the anisotropic case by using the powerful "norm theorem" of Knebusch [K1], [K2] .

Theorem 4. Suppose $q$ is anisotropic, and $\sigma$ is a neighbor to the Pfister form $\varphi$ (i.e., $\sigma<\varphi$ and $\operatorname{dim} \sigma>1 / 2 \operatorname{dim} \varphi$ ). Then $\sigma<\operatorname{Sim}(q)$ implies $\varphi \mid q$. 
No examples of maximal subspaces of similarities have yet been found which do not come from Pfister factors. More precisely, we ask the following question, which is the converse of Theorem 3.

Conjecture. Let $q$ be a quadratic form of dimension $n=2^{r} \cdot t$, where $t$ is odd, and suppose there is a form $\sigma$ with $\sigma<\operatorname{Sim}(q)$ and $\operatorname{dim} \sigma=\rho(n)$. Does $q$ have an $r$-fold Pfister factor?

The decomposition theorem [M], [S] for Clifford algebra representations reduces this question to the case $n=2^{r}$. The conjecture is easily seen to be true when $r \leqslant 3$, and Wadsworth has settled the cases $r=4,5$ by the function field methods. For $r \geqslant 6$, the conjecture has been settled for a certain class of fields (which includes global fields), but it is unknown in general.

\section{REFERENCES} 477-488.

[G1]. M. R. Gabel, Generic orthogonal stably free projectives, J. Algebra 29 (1974),

[G2]. A. V. Geramita and N. J. Pullman, $A$ theorem of Hurwitz and Radon and orthogonal projective modules, Proc. Amer. Math. Soc. 42 (1974), 51-56. MR 48 \#11090.

[G3]. A. V. Geramita and J. S. Wallis, Orthogonal designs, IV, Queen's Mathematical Preprint no. 1974-11, Queen's University, Kingston, Ontario.

[H]. A. Hurwitz, Über.die Komposition der quadratischen Formen, Math. Ann. 88 (1923), 1-25.

[J]. N. Jacobson, Composition algebras and their automorphisms, Rend Circ. Mat. Palermo (2) 7 (1958), 55-80. MR 21 \#66.

[K1]. M. Knebusch, Specializations of quadratic and symmetric bilinear forms, and a norm theorem, Acta. Arith. 24 (1973), 279-299.

[K2]. , Generic splitting of quadratic forms (to appear). 1973.

[L]. T.-Y. Lam, The algebraic theory of quadratic forms, Benjamin, New York,

[M]. A. W. McEvett, Forms over semisimple algebras with involution, J. Algebra 12 (1969), 105-113. MR 43 \#244. pear).

[S]. D. B. Shapiro, Cancellation of semisimple hermitian pairings, J. Algebra (to ap-

[W]. J. A. Wolf, Geodesic spheres in Grassmann manifolds, Illinois J. Math. 7 (1963), 425-446. MR 27 \#6220.

DEPARTMENT OF MATHEMATICS, OHIO STATE UNIVERSITY, COLUMBUS, OHIO 43210 